\title{
Huntington disease alters early neurodevelopment
}

In HD carrier
fetuses, a
smaller pro-
portion of
progenitor
cells were
undergoing
mitosis than
in the control
fetuses

Huntington disease (HD) can alter human neurodevelopment as early as 13 weeks' gestation, according to a new study in Science. The new findings build on previous work in animal models and suggest that disease-modifying therapeutics for HD would need to be administered well before symptoms appear.

$\mathrm{HD}$ is a neurodegenerative disease caused by a mutation in the gene encoding huntingtin (HTT). The symptoms of HD usually begin in mid to late adulthood, but evidence from mouse models suggests that the mutated form of HTT (mHTT) affects neurodevelopment. The new study investigated the effects of mHTT on early brain development in humans.

"HD mutation carriers use prenatal genetic testing to avoid passing on the HD mutation to their children, and in the case of an unfavourable result, couples can choose to terminate a pregnancy and

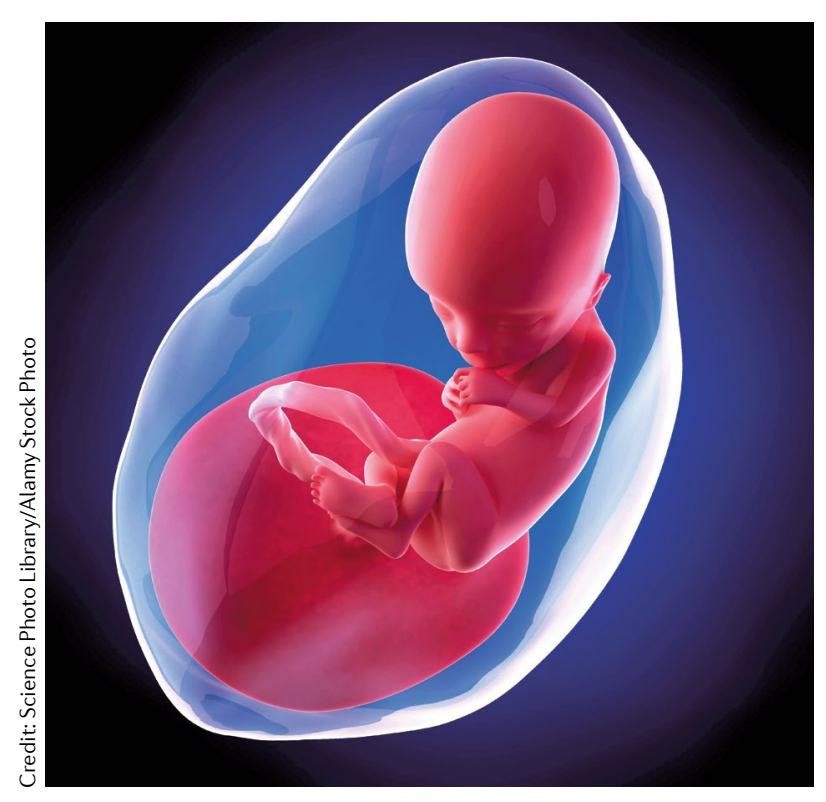

to donate fetal tissue for research," says Sandrine Humbert, who led the study with Alexandra Durr. "We had the opportunity to study intact cortical tissues from four HD mutation carrier fetuses and four healthy control fetuses from pregnancies that were terminated for reasons other than $\mathrm{HD}$ at week 13 of gestation."

At this stage of development, progenitor cells in the ventricular zone are extending processes towards the apical and basal surfaces of the neuroepithelial wall. Neighbouring progenitor cells are linked together by junctional complexes contained within the end-feet, thus sealing the epithelium. These progenitor cells will eventually become cortical neurons that project to the striatum.

The researchers stained the fetal tissue with an antibody that recognized HTT and mHTT. In HD carrier fetuses, this staining was concentrated at the apical end-feet of the progenitor cells, whereas in control fetuses the staining was more widespread. The researchers used two mouse models, one expressing a tagged form of HTT and one expressing a tagged form of $\mathrm{mHTT}$, to investigate this phenomenon further. Compared with HTT, mHTT localized more to the apical surface of the ventricular zone and less to the basal region, indicating that mHTT is mislocalized in these progenitor cells.

One of the main functions of HTT is to transport vesicles, and the researchers stained the human fetal tissue with antibodies for markers of the endosomal pathway. In control fetuses, HTT partially colocalized with several markers of this pathway. However, in HD carrier fetuses,
HTT strongly colocalized with these markers, suggesting that mHTT can alter endosomal trafficking early in development.

The researchers also used immunohistochemistry to detect junctional proteins in the progenitor cells. In HD carrier fetuses, the levels of three junctional proteins ZO1, NCAD and $\beta$-catenin - were increased at the apical surface of the ventricular zone and decreased in the basal region compared with control fetuses.

The apical junctional complexes are essential for cell cycle progression, and the researchers stained the fetal tissue with an antibody to phosphohistone 3 , which is a marker of mitosis. In HD carrier fetuses, a smaller proportion of progenitor cells were undergoing mitosis than in the control fetuses, suggesting that HD results in a smaller pool of proliferating cells.

The researchers also stained the fetal tissue with antibodies to the transcription factors TBR2 and PAX6, which are markers of neuronal maturation - progenitor cells expressing TBR2 are further along the neurogenesis pathway than cells expressing PAX6. HD carrier fetuses had a greater proportion of TBR2-expressing progenitor cells than did control fetuses, indicating that $\mathrm{HD}$ results in premature specification of progenitor cells.

"Understanding that early brain development is abnormal in individuals with HD opens the door to future studies that could identify new molecules of interest for treatment or biomarkers," explains Durr. "Although we do not have disease-modifying treatments yet, our study makes it clear that when treatment is available, the earlier it is started, the better."

Sarah Lemprière

ORIGINAL ARTICLE Barnat, M. et al. Huntington's disease alters human neurodevelopment. Science https://doi.org/10.1126/science.aax3338 (2020) RELATED ARTICLE Wood, H. Huntington disease - a neurodevelopmental disorder? Nat. Rev. Neurol. 14, 632-633 (2018) 